\title{
Bone Regeneration of Peri-Implant Defects Using a Collagen Membrane as a Carrier for Recombinant Human Bone Morphogenetic Protein-2
}

\author{
Yoo-Kyung Sun, ${ }^{1}$ Jae-Kook Cha, ${ }^{1}$ Daniel Stefan Thoma, ${ }^{2}$ So-Ra Yoon ${ }^{(D)},{ }^{1}$ \\ Jung-Seok Lee, ${ }^{1}$ Seong-Ho Choi $\mathbb{D}{ }^{1}{ }^{1}$ and Ui-Won Jung $\mathbb{D}^{1}$ \\ ${ }^{1}$ Department of Periodontology, Research Institute for Periodontal Regeneration, College of Dentistry, Yonsei University, \\ Seoul, Republic of Korea \\ ${ }^{2}$ Clinic for Fixed and Removable Prosthodontics and Dental Material Science, University of Zurich, Zurich, Switzerland \\ Correspondence should be addressed to Ui-Won Jung; drjew@yuhs.ac
}

Received 16 January 2018; Accepted 19 April 2018; Published 25 June 2018

Academic Editor: Gilberto Sammartino

Copyright $\odot 2018$ Yoo-Kyung Sun et al. This is an open access article distributed under the Creative Commons Attribution License, which permits unrestricted use, distribution, and reproduction in any medium, provided the original work is properly cited.

\begin{abstract}
This study is designed to determine the effect of collagen membrane (CM) soaked with bone morphogenetic protein-2 (rhBMP-2) for the treatment of peri-implant dehiscence defects. Material and Methods. Three treatment groups were allocated at each defect in 5 dogs: (i) collagenated synthetic bone (OC) and CM soaked with rhBMP-2 (BMP group), (ii) OC and CM soaked with saline (nonBMP group), and (iii) no further treatment (control group). Titanium pins were used to stabilize the membranes in two dogs. Radiographic and histomorphometric analyses were performed 4 weeks later. Results. The median augmented volumes were 4.27 $\mathrm{mm}^{3}, 6.24 \mathrm{~mm}^{3}$, and $2.75 \mathrm{~mm}^{3}$ in the BMP, nonBMP, and control groups, respectively; the corresponding median first bone-toimplant contact (fBIC) distances were $3.25 \mathrm{~mm}, 3.08 \mathrm{~mm}$, and $2.56 \mathrm{~mm}(P>0.05)$. The placement of pins (with the BMP and nonBMP groups pooled) significantly improved bone regeneration: the augmented volumes were $17.60 \mathrm{~mm}^{3}$ with pins and 3.68 $\mathrm{mm}^{3}$ without pins $(P=0.024)$, with corresponding $\mathrm{fBIC}$ distances of $2.25 \mathrm{~mm}$ and $3.31 \mathrm{~mm}$, respectively $(P<0.001)$. Conclusions. The addition of rhBMP-2 to CM failed to improve bone regeneration of peri-implant dehiscence defects compared to using an unsoaked CM after 4 weeks. However, the stabilization of CMs using pins positively influenced the outcomes.
\end{abstract}

\section{Introduction}

Guided bone regeneration (GBR) using collagen membrane $(\mathrm{CM})$ is a well-documented treatment modality for augmenting localized peri-implant bone defects, with many clinical and preclinical studies demonstrating that exposed implant surfaces can be successfully augmented [1-5]. However, this type of resorbable membrane appears to result in insufficient space maintenance, which is reportedly due to the pressure from the covering flap resulting in membrane collapse $[3,6]$. Recent research has focused on techniques and materials to overcome these drawbacks [7-9].

Recombinant human bone morphogenetic protein-2 (rhBMP-2) has well-documented osteogenic properties that significantly improve bone regeneration [10-13]. Recent reviews have also considered rhBMP-2 to be the most promising bioactive molecules for bone regeneration [14-16].

Clinical considerations mean that dental implants need to be placed in prosthetically ideal positions, which often results in buccal dehiscence defects. Several preclinical and clinical studies have evaluated rhBMP-2 in combination with various bone-substitute materials for localized bone regeneration for this type of peri-implant defect $[10,11,17,18]$. RhBMP-2 was combined with the bone-substitute material in all of these studies; although this resulted in successful treatment outcomes and superiority compared to control groups, the outcomes were to some extent controversial and limited by the clinical applicability of soaking bone-substitute materials 
with rhBMP-2. One option would be to use a CM as a carrier for rhBMP-2, since this would potentially be advantageous in being closer to the highly osteogenic periosteum containing abundant mesenchymal cells. In addition, a rapid bone formation on the outer side of the defect might result in a more stable augmented area. Based on this assumption, Chang and colleagues used a CM as a carrier for rhBMP2 for primary horizontal bone augmentation, demonstrating a proof of concept [18]. However, this combination has not previously been evaluated for the clinically more common peri-implant defects.

Therefore, the aim of the present study was to determine the effect of a CM soaked with rhBMP-2 for the treatment of peri-implant dehiscence defects.

\section{Material and Methods}

2.1. Study Design. The present experiments were designed as a controlled preclinical study involving five mongrel dogs. The dogs were aged 12-15 months and a mean body weight of $30 \mathrm{~kg}$. They had no systemic disease and showed a healthy periodontium and intact dentition. The study was performed in accordance with the Animal Care and Use Committee, Yonsei Medical Center, Seoul, Korea (permission no. 20110188).

2.2. Experimental Materials. The following materials were used in the study:

(1) Titanium implants with a sandblasted and acidetched surface (3.8 $\mathrm{mm}$ in diameter and $8 \mathrm{~mm}$ long) (Implantium ${ }^{\circledR}$, Dentium, Seoul, Korea).

(2) A collagenated synthetic bone (OC; OSTEON ${ }^{\mathrm{TM}} \mathrm{Col}-$ lagen, Dentium) consisting of a particulate bonesubstitute material [70\% hydroxyapatite (HA) and $30 \% \beta$-tricalcium phosphate] and a type I collagen.

(3) A resorbable CM containing HA particles (HA collagen membrane, GENOSS, Suwon, Korea).

(4) rhBMP-2 (Cowellmedi, Busan, Korea) at a concentration of $0.5 \mathrm{mg} / \mathrm{ml}$, which was obtained by reconstituting and diluting rhBMP-2 in a buffer solution. The HA-containing CMs were soaked in $0.2 \mathrm{ml}$ of rhBMP2 solution for 15 minutes at room temperature.

2.3. Surgical Procedures. Oral prophylaxis was applied to all dogs prior to the surgical intervention. Details of the surgical procedures are available elsewhere [19]. In brief, general anesthesia as well as local infiltration anesthesia at the surgical sites was applied. Crevicular incisions were then made from the second premolar to the first molar, and two vertical incisions were made on the buccal gingiva. Following hemisectioning, the second, third, and fourth premolars and the first molar were extracted on one side of the mandible. The buccal bone plates were removed, resulting in an acute defect with dimensions of $5 \mathrm{~mm}$ (apico-coronal width) by $5 \mathrm{~mm}$ (bucco-oral depth) and extending from the second to the fourth premolar. Primary wound closure was then performed. The sutures were removed 10 days later.
2.4. Implant Placement and Guided Bone Regeneration. Twelve weeks later, dental implants were placed and simultaneous GBR was performed (Figure 1). Following flap reflection, the healed ridge was flattened and three implants were placed with their platforms flush with the lingual bone crest. This resulted in a peri-implant dehiscence defect with a height of $3 \mathrm{~mm}$ at the buccal aspect (Figure 2(a)). The cortical bone plate was perforated in the vicinity of the implants, and GBR was performed. The following three treatment modalities were applied (Figure 2(c)):

(1) OC and a CM containing HA particles soaked with rhBMP-2 (BMP group).

(2) OC and a CM containing HA particles soaked with sterile saline (nonBMP group).

(3) No GBR (control group).

The control group was always located at the center implant site to minimize the influence of the BMP molecule on adjacent groups, while the BMP and nonBMP groups were allocated to the mesial or distal implant sites randomly.

In group BMP, the OC was grafted on the peri-implant defect and covered with a CM containing HA particles soaked with rhBMP-2. In group nonBMP, the OC was grafted on the peri-implant defect and then covered with a CM containing HA particles soaked with saline. No overaugmentation was attempted in either of these groups. In two of the five dogs, two titanium pins (Frios ${ }^{\circledR}$ membrane tacks, DENTSPLY Implants, Mannheim, Germany) were used to stabilize the membranes in the BMP and nonBMP groups, while no fixation pins were applied in the remaining three dogs. In addition, the CM was perforated on top of the implant and immobilized by a cover screw. No graft material or membrane was used on the control group.

Periosteal releasing incisions were subsequently made and primary wound closure was achieved using a resorbable suture material (Monosyn ${ }^{\circledR} 4.0$ Glyconate Monofilament, B. Braun, Tuttlingen, Germany). The sutures were removed 10 days later. The dogs were sacrificed by an overdose of sodium pentobarbital 4 weeks after implant placement and GBR surgery.

2.5. Radiographic Analysis. The specimens were scanned using micro-CT (SkyScan 1072, SkyScan, Aartselaar, Belgium) and the total augmented volume (TAV, $\mathrm{mm}^{3}$ ) was measured, which represented the regenerated tissue surrounding the implant. Mineralized tissue was considered to be indicated in the images by grayscale values from 39 to 52 (defined as radiopaque tissue). The lower border of the TAV was located $3 \mathrm{~mm}$ below the implant platform, and the coronal border was defined by the most-coronal location of radiopaque tissue. The buccolingual extension of the TAV ranged from the center of the implant to the most-buccal radiopaque tissue (at an angle of $90^{\circ}$ to the implant surface). The mesiodistal borders of the TAV were confined by vertical lines $7 \mathrm{~mm}$ from the center of the implant surface (Figure 3). The implant itself was excluded from the TAV.

Cross-sectional images of each group are presented in Figure 4 . The total augmented materials were painted using 


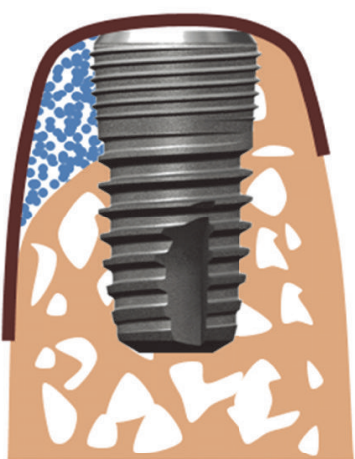

BMP group

: a\#tsas Bone substitute

CM soaked with rhBMP-2

(a)

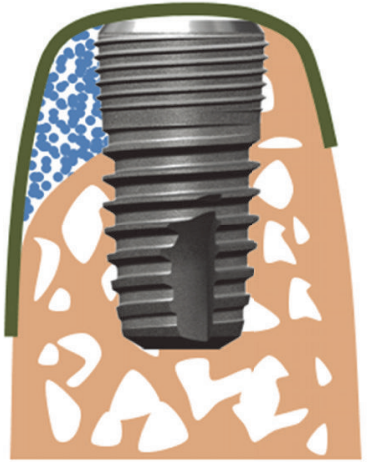

nonBMP group

atiscos

.
Bone substitute

CM soaked with saline

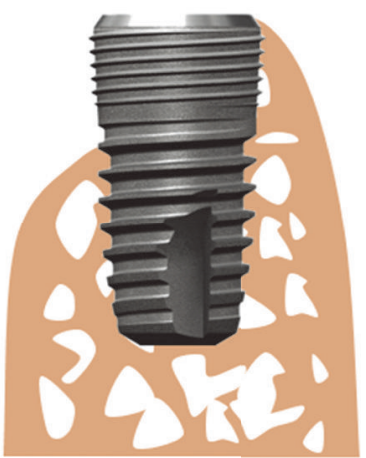

control group

(b)

(c)

FIGURE 1: Schematic drawings of the surgery design: (a) BMP group, (b) nonBMP group, and (c) control group. Blue circles, bone-substitute materials; dark-red line, CM soaked with rhBMP-2; green line, CM soaked with saline; BMP group, cylinder-type bone-substitute material covered by CM soaked with rhBMP-2; nonBMP group, cylinder-type bone-substitute material covered by CM soaked with saline; control group, no further treatment.

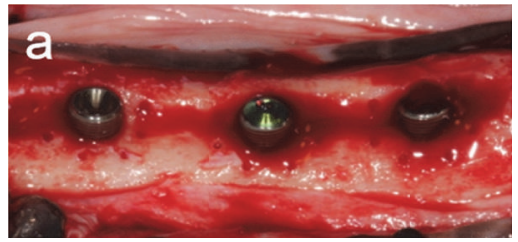

(a)

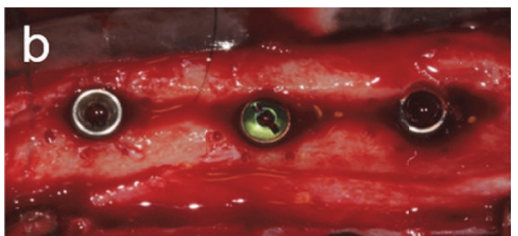

(b)

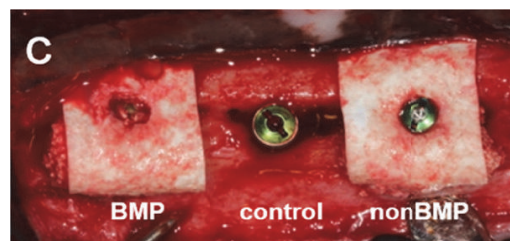

(c)

FIGURE 2: Clinical photographs of the bone augmentation procedure applied to peri-implant dehiscence defects: (a) buccal and (b) occlusal views after implant placement and (c) GBR treatment performed on dehiscence defects according to group assignment. The control group was placed at the center implant site, and the BMP and nonBMP groups were randomly allocated to the mesial and distal implant sites.

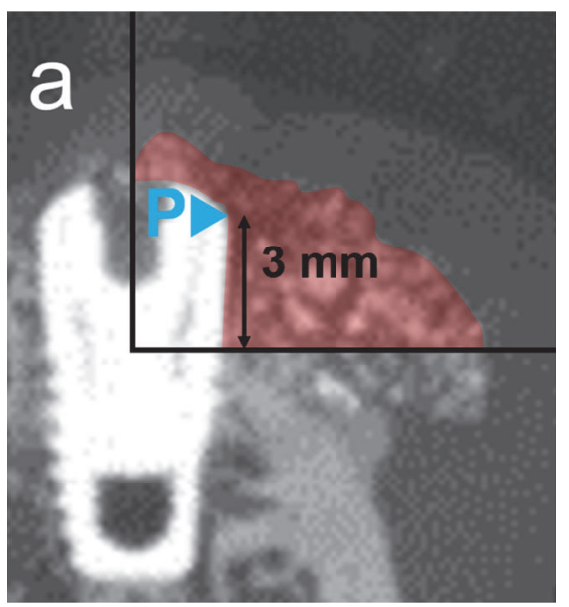

(a)

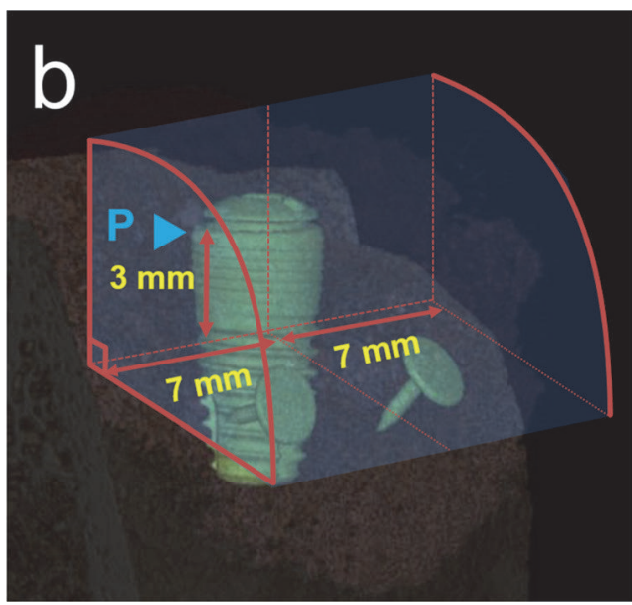

(b)

FIgURE 3: Schematic configuration of the TAV $\left(\mathrm{mm}^{3}\right)$. (a) The lower border of the TAV was located $3 \mathrm{~mm}$ below the implant platform. The coronal border was defined by the most-coronal location of radiopaque tissue. The buccolingual extension of the TAV ranged from the center of the implant to the most-buccal radiopaque tissue (at an angle of $90^{\circ}$ to the implant surface). (b) The mesiodistal borders of the TAV were confined by vertical lines $7 \mathrm{~mm}$ from the center of the implant surface. The implant itself was excluded from the TAV. P, implant platform. 


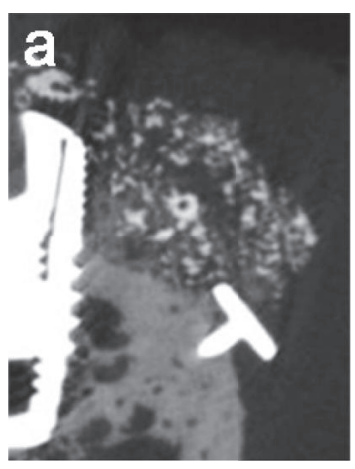

(a)

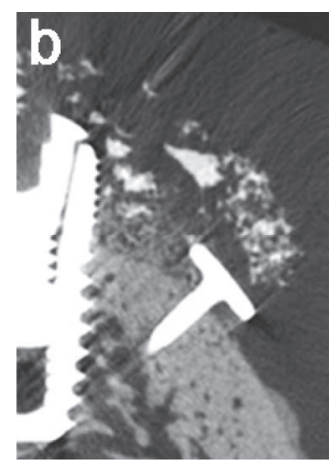

(b)

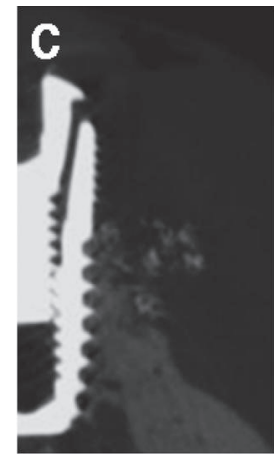

(c)

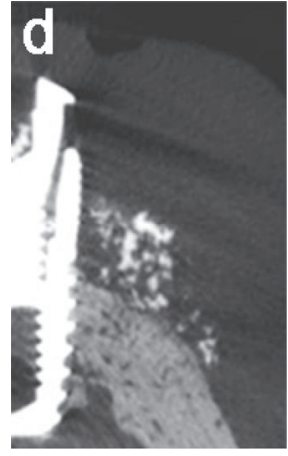

(d)

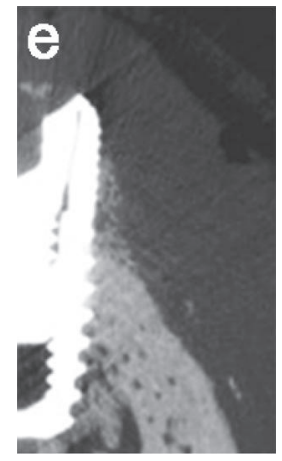

(e)

FIGURE 4: Cross-sectional radiographic images demonstrating variations both between and within the groups: (a) BMP group with pins, (b) nonBMP group with pins, (c) BMP group without pins, (d) nonBMP group without pins, and (e) control group. In the BMP and nonBMP groups, the shape of the augmented region appeared to be predominantly influenced by the use of pins.

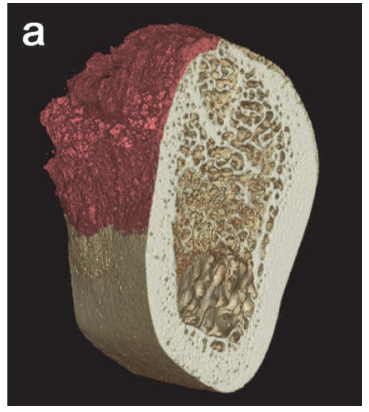

(a)

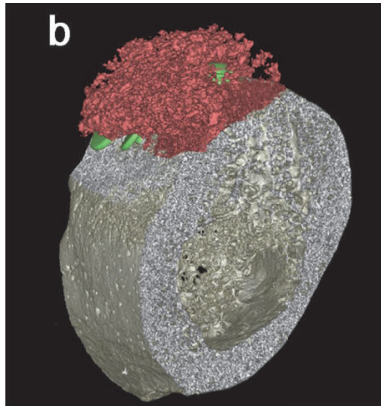

(b)

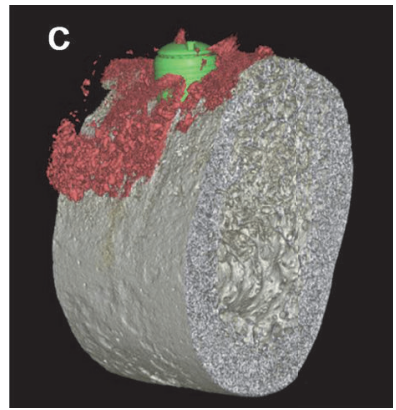

(c)

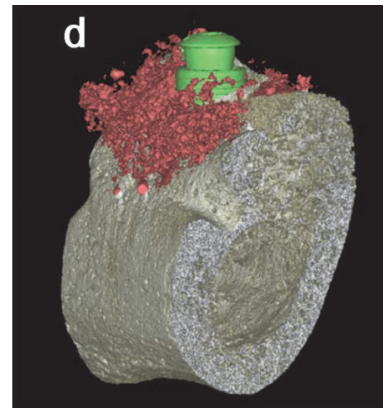

(d)

FIgURE 5: Three-dimensional reconstructed color-coded images of the total augmented area: (a) BMP group with pins, (b) nonBMP group with pins, (c) BMP group without pins, and (d) nonBMP group without pins. No remaining peri-implant defects were observed in the specimens with pins, in contrast with the sites without pins and control sites without GBR.

OnDemand 3D software (Cybermed, Seoul, Korea) to facilitate identification of the bone regeneration (Figure 5).

2.6. Histologic Analysis. Block specimens were harvested that included the implants and grafted sites with surrounding hard and soft tissues, and they were fixed in $10 \%$ neutral buffered formalin for 10 days. The specimens were then trimmed and dehydrated in ethanol before being embedded in methyl methacrylate. Specimens were cut in the center of the implant sites in a buccolingual plane and stained with hematoxylineosin and Masson's trichrome. The final thickness of the sections was $20 \mu \mathrm{m}$.

2.7. Descriptive Histology. The histology sections were examined under a light microscope (BX-50, Olympus Optical, Tokyo, Japan) to identify relevant structures such as the implants, new bone formation, bone-substitute material, nonmineralized tissue, and the remaining peri-implant defect.

2.8. Histomorphometric Analysis. Histomorphometric measurements were made at the buccal aspect of all implants.
An image-analysis program (Adobe Photoshop CS6, Adobe Systems, San Jose, CA, USA) was used to assess the following landmarks: implant platform $(\mathrm{P})$, the first bone-to-implant contact (B), and the most-coronal buccal bone (BC) (Figure 6).

2.8.1. Linear Measurements. The following linear measurements were made:

(1) Distance between the implant platform $(\mathrm{P})$ and the first bone-to-implant contact (B) (fBIC, $\mathrm{mm}$ ).

(2) Vertical distance between the most-coronal buccal bone $(\mathrm{BC})$ and the implant platform $(\mathrm{P})(\mathrm{P}-\mathrm{BC}, \mathrm{mm})$.

(3) The bone-to-implant contact (BIC, \%), measured along the implant surface between the implant platform $(\mathrm{P})$ and extending $4 \mathrm{~mm}$ apically.

2.8.2. Area/Surface Measurements. A rectangular area of interest (AOI) was defined on the buccal side of the implants and included the following dimensions: coronal (implant platform), apical (4 mm apically toward the implant platform), and horizontal ( $2 \mathrm{~mm}$ from the implant surface). 


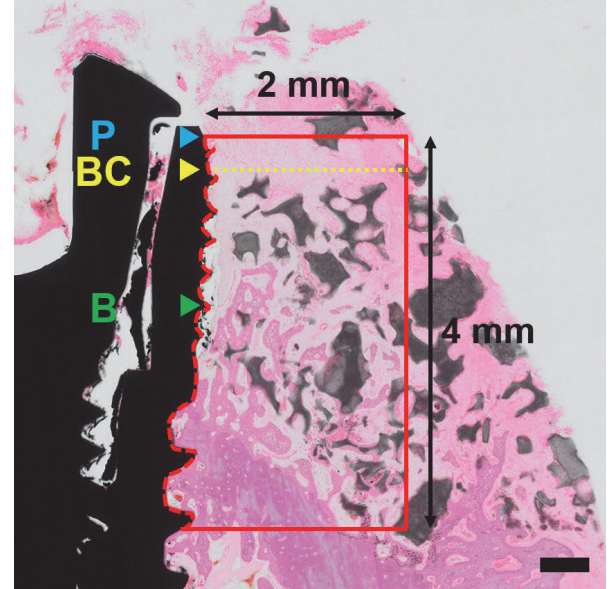

FIGURE 6: Schematic configuration for the histomorphometric analysis. P, implant platform; BC, most-coronal buccal bone; B, first bone-to-implant contact; red box, AOI. Scale bar $=500 \mu \mathrm{m}$.

The ratios of the following outcomes to the AOI area were measured:

(1) Newly formed bone (NB, \%).

(2) Residual bone-substitute material (RBS, \%).

(3) Nonmineralized tissue (NMT, \%).

2.9. Statistical Analysis. Mean and standard deviation values were calculated in each group. One-way analysis of variance and the Bonferroni post hoc test were used to assess the clinical benefit of soaking the CM with the rhBMP-2 solution. The independent $t$-test was used to identify differences among groups related to the use of pins. The cutoff for statistical significance was $P<0.05$.

\section{Results}

3.1. Clinical Findings. All of the dogs remained healthy, with no wound dehiscence or membrane exposure being detected throughout the experiments. All of the obtained samples were included in the analyses.

3.2. Radiographic Analysis. The cross-sectional images demonstrated variations both between and within the groups (Figure 4). In the BMP and nonBMP groups, the shape of the augmented region appeared to be predominantly influenced by the use of pins. No remaining peri-implant defects were observed in the specimens with pins, in contrast with the sites without pins and control sites without GBR (Figure 5).

The median TAV values were $4.27 \mathrm{~mm}^{3}, 6.24 \mathrm{~mm}^{3}$, and $2.75 \mathrm{~mm}^{3}$ in the BMP, nonBMP, and control groups, respectively (all $P>0.05$ ) (Table 1 ). When the groups were divided according to the presence or absence of fixation pins, the median TAV was significantly higher in the group with pins $\left(17.60 \mathrm{~mm}^{3}\right)$ than in that without pins $\left(3.68 \mathrm{~mm}^{3}\right)(P=$ 0.024) (Table 2).
TABle 1: Measured the total augmented volume (TAV) values. $P$ value for intergroup comparison $=0.376 . n=$ number of sites $/$ dogs.

\begin{tabular}{lc}
\hline Group & TAV $\left(\mathrm{mm}^{3}\right)$ \\
\hline BMP group & $4.27(3.08-18.23)$ \\
with pins $(n=2)$ & $20.87(19.55-22.20)$ \\
without pins $(n=3)$ & $3.08(1.68-3.68)$ \\
nonBMP group & $6.24(5.71-7.52)$ \\
with pins $(n=2)$ & $12.25(9.88-14.62)$ \\
without pins $(n=3)$ & $5.71(3.15-5.98)$ \\
Control group & $2.75(1.98-4.80)$ \\
\hline
\end{tabular}

Data are median (interquartile range) values.

3.3. Histologic Observations and Histomorphometric Analysis. In the two GBR groups (i.e., BMP and nonBMP), bone formation was generally observed along the implant surfaces (Figure 7). However, defect resolution was not consistent, with some specimens in both groups exhibiting complete regeneration and others with remaining peri-implant bone defects. In control sites, the size of the peri-implant defects appeared to be similar to that prior to augmentation. Complete resolution of the dehiscence defects was consistently observed in the histology specimens of the BMP and nonBMP groups using pins, with new bone having formed at the apical border of the bone defect and around the bone-substitute particles. In contrast, the positioning of the bone-substitute particles was disrupted and bone regeneration did not occur uniformly in the group without pins.

The median fBIC distances were $3.25 \mathrm{~mm}, 3.08 \mathrm{~mm}$, and $2.56 \mathrm{~mm}$ in the BMP, nonBMP, and control groups, respectively, with corresponding median BIC values of $11.90 \%$, $18.24 \%$, and $21.96 \%$.

Within the AOI, the median NBs were $12.84 \%, 8.06 \%$, and $21.75 \%$ in the BMP, nonBMP, and control groups, respectively. The median RBS was $1.31 \%$ in the BMP group and $12.43 \%$ in the nonBMP group. There were no significant intergroup differences $(P>0.05)$. All of the data are reported in Table 3 .

All of the measured histomorphometric and radiographic values (except for RBS in histomorphometric analyses) differed significantly with the presence or absence of pins when 10 specimens were divided according to the use of pins (Table 2). The median NB was $37.03 \%$ with pins and $4.87 \%$ without pins in the BMP group and $47.18 \%$ with pins and $7.90 \%$ without pins in the nonBMP group. BIC was higher in both the BMP and nonBMP groups with pins $(40.38 \%$ and $33.19 \%$, respectively) than without pins (10.49\% and $10.69 \%$, respectively) (Table 3 ).

\section{Discussion}

This study has revealed that (i) the addition of rhBMP2 to a CM does not significantly improve peri-implant bone regeneration and (ii) fixation of the CM using pins significantly increases bone regeneration compared to using a CM without pins.

This study was designed to evaluate the usefulness of a $\mathrm{CM}$ as a carrier for rhBMP-2 for guided bone regeneration at 


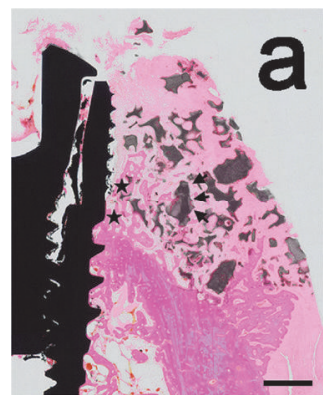

(a)

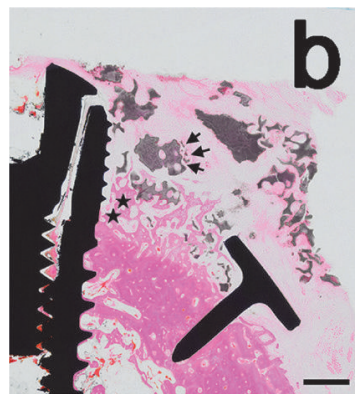

(b)

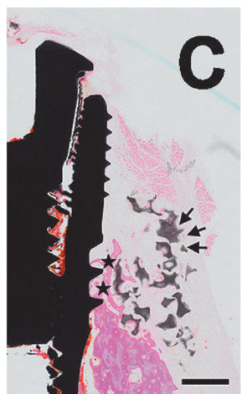

(c)

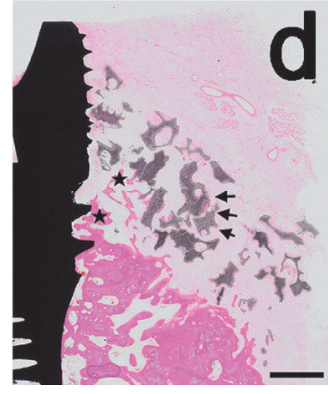

(d)

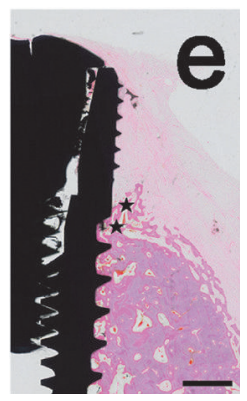

(e)

FIGURE 7: Histologic views presenting the results of GBR: (a) BMP group with pins, (b) nonBMP group with pins, (c) BMP group without pins, (d) nonBMP group without pins, and (e) control group. The results for defect resolution were not consistent, as demonstrated by some specimens in both the BMP and nonBMP groups exhibiting complete regeneration and others with remaining peri-implant bone defects. Complete resolution of the dehiscence defects was consistently observed in the histology specimens of the BMP and nonBMP groups using pins, while the positioning of bone-substitute particles was disrupted and bone regeneration did not occur uniformly in the group without pins. In the control sites, the size of the peri-implant defects appeared to be similar to the situation prior to augmentation. Asterisk, newly formed bone; arrow, residual bone-substitute material. Scale bar $=1 \mathrm{~mm}$.

TABLE 2: Radiographic and histomorphometric outcomes, with data for the BMP and nonBMP groups pooled according to the use of pins.

\begin{tabular}{|c|c|c|c|c|}
\hline & & With pins $(n=4)$ & Without pins $(n=6)$ & $P$ \\
\hline Radiographic volume & TAV, $\mathrm{mm}^{3}$ & $17.60(7.52-23.52)$ & $3.68(0.27-5.71)$ & 0.024 \\
\hline \multirow{3}{*}{ Linear measurements } & BIC, $\%$ & $40.38(18.24-48.15)$ & $10.59(2.61-26.12)$ & 0.027 \\
\hline & $\mathrm{fBIC}, \mathrm{mm}$ & $2.25(1.95-2.38)$ & $3.31(3.08-0.87)$ & $<0.001$ \\
\hline & $\mathrm{P}-\mathrm{BC}, \mathrm{mm}$ & $0.59(0.00-1.27)$ & $2.91(2.51-3.44)$ & 0.001 \\
\hline \multirow{3}{*}{ Measurements in the AOI } & $\mathrm{NB}, \%$ & $42.54(28.69-54.66)$ & $6.56(3.40-12.84)$ & 0.006 \\
\hline & RBS, \% & $8.03(0.00-19.01)$ & $6.86(0.00-23.66)$ & 0.908 \\
\hline & NMT, \% & $53.46(32.92-56.66)$ & $84.94(68.29-95.29)$ & 0.002 \\
\hline
\end{tabular}

Data are median (interquartile range) values.

$n=$ number of sites/dogs. TAV $\left(\mathrm{mm}^{3}\right)=$ the total augmented volume; BIC $(\%)=$ the bone-to-implant contact; fBIC $(\mathrm{mm})=$ distance between the implant platform and the first bone-to-implant contact; P-BC $(\mathrm{mm})=$ vertical distance between the most-coronal buccal bone and the implant platform; $\mathrm{NB}(\%)=$ newly formed bone; RBS (\%) = residual bone-substitute material; NMT $(\%)=$ nonmineralized tissue.

TABLE 3: Histomorphometric measurements.

\begin{tabular}{|c|c|c|c|c|c|c|}
\hline \multirow[b]{2}{*}{ Group } & \multicolumn{3}{|c|}{ Linear measurements } & \multicolumn{3}{|c|}{ Measurements in the AOI } \\
\hline & $\mathrm{BIC}, \%$ & $\mathrm{fBIC}, \mathrm{mm}$ & $\mathrm{P}-\mathrm{BC}, \mathrm{mm}$ & $\mathrm{NB}, \%$ & RBS, \% & NMT, \% \\
\hline BMP group & $\begin{array}{c}11.90 \\
(10.49-34.65)\end{array}$ & $\begin{array}{c}3.25 \\
(2.29-3.36)\end{array}$ & $\begin{array}{c}2.64 \\
(1.27-2.75)\end{array}$ & $\begin{array}{c}12.84 \\
(4.87-28.69)\end{array}$ & $\begin{array}{c}1.31 \\
(0.00-12.42)\end{array}$ & $\begin{array}{c}82.71 \\
(54.63-87.16)\end{array}$ \\
\hline $\begin{array}{l}\text { with pins } \\
(n=2)\end{array}$ & $\begin{array}{c}40.38 \\
(37.52-43.25)\end{array}$ & $\begin{array}{c}2.25 \\
(2.23-2.27)\end{array}$ & $\begin{array}{c}0.64 \\
(0.32-0.95)\end{array}$ & $\begin{array}{c}37.03 \\
(32.86-41.20)\end{array}$ & $\begin{array}{c}9.51 \\
(4.75-14.26)\end{array}$ & $\begin{array}{c}53.46 \\
(52.88-54.05)\end{array}$ \\
\hline $\begin{array}{l}\text { without pins } \\
(n=3)\end{array}$ & $\begin{array}{c}10.49 \\
(6.55-11.20)\end{array}$ & $\begin{array}{c}3.36 \\
(3.31-3.62)\end{array}$ & $\begin{array}{c}2.75 \\
(2.70-3.04)\end{array}$ & $\begin{array}{c}4.87 \\
(4.14-8.86)\end{array}$ & $\begin{array}{c}1.31 \\
(0.65-6.86)\end{array}$ & $\begin{array}{c}87.16 \\
(84.94-91.22)\end{array}$ \\
\hline nonBMP group & $\begin{array}{c}18.24 \\
(10.69-26.12)\end{array}$ & $\begin{array}{c}3.08 \\
(2.38-3.17)\end{array}$ & $\begin{array}{c}2.51 \\
(0.65-3.06)\end{array}$ & $\begin{array}{c}8.06 \\
(7.90-39.71)\end{array}$ & $\begin{array}{c}12.43 \\
(3.63-19.56)\end{array}$ & $\begin{array}{c}68.29 \\
(56.66-75.23)\end{array}$ \\
\hline $\begin{array}{l}\text { with pins } \\
(n=2)\end{array}$ & $\begin{array}{c}33.19 \\
(25.72-40.67)\end{array}$ & $\begin{array}{c}2.17 \\
(2.06-2.27)\end{array}$ & $\begin{array}{c}0.59 \\
(0.56-0.62)\end{array}$ & $\begin{array}{c}47.18 \\
(43.45-50.92)\end{array}$ & $\begin{array}{c}8.03 \\
(5.83-10.23)\end{array}$ & $\begin{array}{c}44.79 \\
(38.85-50.73)\end{array}$ \\
\hline $\begin{array}{l}\text { without pins } \\
(n=3)\end{array}$ & $\begin{array}{c}10.69 \\
(10.33-18.41)\end{array}$ & $\begin{array}{c}3.17 \\
(3.13-3.31)\end{array}$ & $\begin{array}{c}3.06 \\
(2.79-3.25)\end{array}$ & $\begin{array}{c}7.90 \\
(6.56-7.98)\end{array}$ & $\begin{array}{c}19.56 \\
(9.78-21.61)\end{array}$ & $\begin{array}{c}75.2 \\
(71.76-83.66)\end{array}$ \\
\hline Control group & $\begin{array}{c}21.96 \\
(8.16-5.04)\end{array}$ & $\begin{array}{c}2.56 \\
(2.55-3.74)\end{array}$ & $\begin{array}{c}2.04 \\
(1.60-3.56)\end{array}$ & $\begin{array}{c}21.75 \\
(3.19-22.91)\end{array}$ & 0.00 & $\begin{array}{c}78.25 \\
(77.09-96.81)\end{array}$ \\
\hline$P$ & 0.873 & 0.664 & 0.679 & 0.550 & 0.313 & 0.152 \\
\hline
\end{tabular}

Data are median (interquartile range) values.

$n=$ number of sites/dogs.

None of the outcome measured differed significantly between the three groups $(P>0.05)$. 
peri-implant dehiscence defects. Previous experiments that employed a similar protocol using animals and $30-\mathrm{mm}$ periimplant defects demonstrated that this model can serve as a valid alternative to clinical studies [20,21].

Various preclinical models have demonstrated that rhBMP-2 improves bone regeneration by accelerating osteogenesis predominantly at the early stage of wound healing $[22,23]$. In addition, the healing time is reportedly twofold shorter in dogs than in humans [24], and so earlier observation periods were established in this present study when evaluating the efficacy of rhBMP-2 compared to the previous experiments. In the present study, the bone formation at 4 weeks was greater in the GBR groups (by up to $47 \%$ ) than at the untreated control sites $(22 \%)$. This is in line with the reporting greater bone formation for GBR groups compared to the control sites, although those results were for longer healing periods of 8 and 16 weeks [21]. However, the addition of rhBMP-2 to a CM in the present study was not beneficial to bone regeneration compared to a CM soaked in saline at the early stage of healing of 4 weeks.

The other main difference between these two studies-apart from the healing period-was in the type of carrier material used. Various carrier materials have been used for rhBMP-2 [25-27]. It was speculated that using a $\mathrm{CM}$ as a carrier for rhBMP-2 could enhance the osteoinductivity of the periosteum due to the close proximity of the membrane and the periosteum. It was also speculated that an rhBMP-2 carrier in such a location would result in faster bone regeneration at the borders of the defect area, thereby ideally leading to shell-like bone formation, which could further compensate the disadvantages of a resorbable nonspace-maintaining membrane. However, this outcome was not observed in the present study, with no shell-like bone formation observed in any of the samples. In a preliminary study that used a GBR protocol and materials similar to those in the present study [18], histomorphometric analyses revealed that new bone formed closer to the membrane in the group with an rhBMP-2-loaded membrane than in the group with rhBMP-2 loaded on the bone-substitute material. This was observed as the new bone formed directly underneath the membrane in the group with rhBMP-2 loaded membrane. The differences in the observed bone regeneration pattern between these two studies might have been due to different healing periods ( 8 weeks versus 4 weeks), the placement of implants versus GBR alone, and the use of fixation pins.

Stability of the surgical site and space maintenance are considered to be essential for successful bone regeneration. The stabilization of a GBR site appears to be the critical factor governing the amount of bone formation. In a clinical study, it was found that bone formation was superior when using fixation pins [4]. This was subsequently supported by two in vitro studies that evaluated the effect of wound closure on the stability of GBR sites using various material combinations $[6,28]$, which found that the bone-substitute material moved apically upon wound closure. However, CMs were used at all sites, and it is well known that this type of membrane is weak mechanically and so may not be able to resist compressive forces. This can result in collapse of the membrane and the above-mentioned displacement of bone-substitute material. However, the studies have demonstrated that applying additional fixation pins can reduce the membrane displacement by $50 \%$ at the level of the implant shoulder. The clinical recommendation was to add fixation pins when CMs are used in combination with particulate graft materials [6]. Fixation pins were used in two of the five dogs in the present study. The failure to standardize the experimental method was due to not being possible to fix the pins on thick, rigid cortical bone without deformation. The results demonstrate the beneficial bone formation at sites where pins are used. However, it could be considered to use alternative fixation materials, such as miniscrews which have better strength than pins in case of performing GBR on a rigid bony plate.

Apart from the fixation pins, more-rigid barrier membranes [29] or more-stable grafting materials provide further advantages in stabilizing the augmented site [30]. This was implemented in the present study by combining a cylindrical type of synthetic bone-substitute material with a type I collagen matrix and HA-coated CMs. This bonesubstitute material incorporating a collagen matrix was considered to resist compressive forces and to support the augmented ridge volume [31]. An in vitro study using a similar membrane coated with HA demonstrated a significantly enhanced chemical stability and an improved mechanical structure of the membrane [32]. Moreover, the cross-linked chemical structure stiffened the CM and thereby reduced the risk of collapse [33]. Such a membrane theoretically exhibits all the characteristics necessary to support space maintenance. However, in clinical experiments the increased stiffness resulted in major difficulties in handling the membrane and applying it properly to the defect site, which meant that displacement of the graft material could not be avoided.

The outcomes of this study are limited by several factors, including the relatively small number of experimental animals, the use of pins in only two of the five dogs, the handling difficulties with the membrane resulting in suboptimal clinical outcomes, and the short observation period. Further studies involving larger numbers of animals and appropriate statistical analyses are required to confirm the results of this study.

\section{Conclusion}

The use of rhBMP-2 soaked on a CM as a carrier material did not result in superior bone formation compared to control sites without rhBMP-2. However, the use of fixation pins to stabilize the CMs did exert a positive effect on peri-implant bone regeneration.

\section{Data Availability}

The data used to support the findings of this study are available from the corresponding author upon request. 


\section{Disclosure}

This article was presented at the 26th Annual Scientific Meeting of the European Association for Osseointegration (EAO), Madrid, October 5-7, 2017.

\section{Conflicts of Interest}

The authors have no conflicts of interest related to this article to report.

\section{Authors' Contributions}

Yoo-Kyung Sun and Jae-Kook Cha contributed equally to this study.

\section{Acknowledgments}

This work was supported by the National Research Foundation of Korea (NRF) grant funded by the Korea Government (Ministry of Science, ICT \& Future Planning) (no. NRF2017R1A2B2002537).

\section{References}

[1] D. Brocard, P. Barthet, E. Baysse et al., "A multicenter report on 1,022 consecutively placed ITI implants: a 7-year longitudinal study," The International Journal of Oral \& Maxillofacial Implants, vol. 15, no. 5, pp. 691-700, 2000.

[2] N. U. Zitzmann, R. Naef, and P. Schärer, "Resorbable versus nonresorbable membranes in combination with Bio-Oss for guided bone regeneration," International Journal of Oral \& Maxillofacial Implants, vol. 12, no. 6, pp. 844-852, 1997.

[3] T. J. Oh, S. J. Meraw, E. J. Lee, W. V. Giannobile, and H. L. Wang, "Comparative analysis of collagen membranes for the treatment of implant dehiscence defects," Clinical Oral Implants Research, vol. 14, no. 1, pp. 80-90, 2003.

[4] L. Carpio, J. Loza, S. Lynch, and R. Genco, "Guided bone regeneration around endosseous implants with anorqanic bovine bone mineral. a randomized controlled trial comparing bioabsorbable versus non-resorbable barriers," Journal of Periodontology, vol. 71, no. 11, pp. 1743-1749, 2000.

[5] J. J. Sevor, R. M. Meffert, and R. Jack Cassingham, "Regeneration of dehisced alveolar bone adjacent to endosseous dental implants utilizing a resorbable collagen membrane: Clinical and histologic results," International Journal of Periodontics and Restorative Dentistry, vol. 13, no. 1, pp. 71-83, 1993.

[6] J. Mir-Mari, H. Wui, R. E. Jung, C. H. F. Hämmerle, and G. I. Benic, "Influence of blinded wound closure on the volume stability of different GBR materials: An in vitro cone-beam computed tomographic examination," Clinical Oral Implants Research, vol. 27, no. 2, pp. 258-265, 2016.

[7] D. S. Thoma, U. W. Jung, J. Y. Park, S. P. Bienz, J. Hüsler, and R. E. Jung, "Bone augmentation at peri-implant dehiscence defects comparing a synthetic polyethylene glycol hydrogel matrix vs. standard guided bone regeneration techniques," Clinical Oral Implants Research, vol. 28, no. 7, pp. e76-e83, 2017.

[8] I. A. Urban, J. L. Lozada, B. Wessing, F. Suarez-Lopez del Amo, and H. L. Wang, "Vertical bone grafting and periosteal vertical mattress suture for the fixation of resorbable membranes and stabilization of particulate grafts in horizontal guided bone regeneration to achieve more predictable results: A technical report," International Journal of Periodontics and Restorative Dentistry, vol. 36, no. 2, pp. 153-159, 2016.

[9] G. Iglhaut, F. Schwarz, M. Gründel, I. Mihatovic, J. Becker, and H. Schliephake, "Shell technique using a rigid resorbable barrier system for localized alveolar ridge augmentation," Clinical Oral Implants Research, vol. 25, no. 2, pp. el49-e154, 2014.

[10] R. E. Jung, R. Glauser, P. Schärer, C. H. F. Hàmmerle, H. F. Sailer, and F. E. Weber, "Effect of rhBMP-2 on guided bone regeneration in humans: a randomized, controlled clinical and histomorphometric study," Clinical Oral Implants Research, vol. 14, no. 5, pp. 556-568, 2003.

[11] R. E. Jung, S. I. Windisch, A. M. Eggenschwiler, D. S. Thoma, F. E. Weber, and C. H. F. Hämmerle, "A randomized-controlled clinical trial evaluating clinical and radiological outcomes after 3 and 5 years of dental implants placed in bone regenerated by means of GBR techniques with or without the addition of BMP2," Clinical Oral Implants Research, vol. 20, no. 7, pp. 660-666, 2009.

[12] J. K. Cha, J. S. Lee, M. S. Kim, S. H. Choi, K. S. Cho, and U. W. Jung, "Sinus augmentation using BMP-2 in a bovine hydroxyapatite/collagen carrier in dogs," Journal of Clinical Periodontology, vol. 41, no. 1, pp. 86-93, 2014.

[13] J. W. Jang, J. H. Yun, K. I. Lee et al., "Osteoinductive activity of biphasic calcium phosphate with different rhBMP-2 doses in rats," Oral Surgery, Oral Medicine, Oral Pathology, Oral Radiology, and Endodontology, vol. 113, no. 4, pp. 480-487, 2012.

[14] P. C. Bessa, M. Casal, and R. L. Reis, "Bone morphogenetic proteins in tissue engineering: the road from laboratory to clinic, part II (BMP delivery)," Journal of Tissue Engineering and Regenerative Medicine, vol. 2, no. 2-3, pp. 81-96, 2008.

[15] H. Cheng, W. Jiang, F. M. Phillips et al., "Osteogenic activity of the fourteen types of human bone morphogenetic proteins (BMPs)," The Journal of Bone \& Joint Surgery, vol. 85, no. 8, pp. 1544-1552, 2003

[16] X. Ji, D. Chen, C. Xu, S. E. Harris, G. R. Mundy, and T. Yoneda, "Patterns of gene expression associated with BMP-2induced osteoblast and adipocyte differentiation of mesenchymal progenitor cell 3T3-F442A," Journal of Bone and Mineral Metabolism, vol. 18, no. 3, pp. 132-139, 2000.

[17] F. Schwarz, D. Rothamel, M. Herten, D. Ferrari, M. Sager, and J. Becker, "Lateral ridge augmentation using particulated or block bone substitutes biocoated with rhGDF-5 and rhBMP-2: an immunohistochemical study in dogs," Clinical Oral Implants Research, vol. 19, no. 7, pp. 642-652, 2008.

[18] Y. Y. Chang, J. S. Lee, M. S. Kim, S. H. Choi, J. K. Chai, and U. W. Jung, "Comparison of collagen membrane and bone substitute as a carrier for rhBMP-2 in lateral onlay graft," Clinical Oral Implants Research, vol. 26, no. 1, pp. e13-e19, 2015.

[19] I. K. Lee, H. C. Lim, J. S. Lee, J. Y. Hong, S. H. Choi, and U. W. Jung, "Layered approach with autogenous bone and bone substitute for ridge augmentation on implant dehiscence defects in dogs," Clinical Oral Implants Research, vol. 27, no. 5, pp. 622628, 2016.

[20] K. Ito, Y. Yamada, R. Ishigaki, K. Nanba, T. Nishida, and S. Sato, "Effects of guided bone regeneration with non-resorbable and bioabsorbable barrier membranes on osseointegration around hydroxyapatite-coated and uncoated threaded titanium dental implants placed into a surgically-created dehiscence type defect in rabbit tibia: a pilot study," Journal of oral science, vol. 43, no. 1, pp. 61-67, 2001. 
[21] D. S. Thoma, J. K. Cha, V. M. Sapata, R. E. Jung, J. Hüsler, and U. W. Jung, "Localized bone regeneration around dental implants using recombinant bone morphogenetic protein-2 and plateletderived growth factor-BB in the canine," Clinical Oral Implants Research, vol. 28, no. 11, pp. 1334-1341, 2017.

[22] J. Y. Hong, M. S. Kim, H. C. Lim, J. S. Lee, S. H. Choi, and U. W. Jung, "A high concentration of recombinant human bone morphogenetic protein-2 induces low-efficacy bone regeneration in sinus augmentation: a histomorphometric analysis in rabbits," Clinical Oral Implants Research, vol. 27, no. 12, pp. e199-e205, 2016.

[23] U. M. E. Wikesjö, M. Qahash, G. Polimeni et al., "Alveolar ridge augmentation using implants coated with recombinant human bone morphogenetic protein-2: histologic observations," Journal of Clinical Periodontology, vol. 35, no. 11, pp. 1001-1010, 2008.

[24] H. Burchardt, "The biology of bone graft repair," Clinical Orthopaedics and Related Research, vol. 174, pp. 28-42, 1983.

[25] M. P. Kelly, O. L. A. Vaughn, and P. A. Anderson, "Systematic review and meta-analysis of recombinant human bone morphogenetic protein-2 in localized alveolar ridge and maxillary sinus augmentation," Journal of Oral and Maxillofacial Surgery, vol. 74, no. 5, pp. 928-939, 2016.

[26] H. Schliephake, "Clinical efficacy of growth factors to enhance tissue repair in oral and maxillofacial reconstruction: a systematic review," Clinical Implant Dentistry and Related Research, vol. 17, no. 2, pp. 247-273, 2015.

[27] C. H. Lai, L. Zhou, Z. L. Wang, H. B. Lu, and Y. Gao, "Use of a collagen membrane loaded with recombinant human bone morphogenetic protein-2 with collagen-binding domain for vertical guided bone regeneration," Journal of Periodontology, vol. 84, no. 7, pp. 950-957, 2013.

[28] J. Mir-Mari, G. I. Benic, E. Valmaseda-Castellón, C. H. F. Hämmerle, and R. E. Jung, "Influence of wound closure on the volume stability of particulate and non-particulate GBR materials: an in vitro cone-beam computed tomographic examination. Part II," Clinical Oral Implants Research, vol. 28, no. 6, pp. 631639, 2017.

[29] N. Naenni, D. Schneider, R. E. Jung, J. Hüsler, C. H. Hämmerle, and D. S. Thoma, "Randomized clinical study assessing two membranes for guided bone regeneration of peri-implant bone defects: clinical and histological outcomes at 6 months," Clinical Oral Implants Research, vol. 28, no. 10, pp. 1309-1317, 2017.

[30] G. I. Benic, D. S. Thoma, F. Muñoz, I. Sanz Martin, R. E. Jung, and C. H. F. Hämmerle, "Guided bone regeneration of periimplant defects with particulated and block xenogenic bone substitutes," Clinical Oral Implants Research, vol. 27, no. 5, pp. 567-576, 2016.

[31] U. W. Jung, J. S. Lee, W. Y. Park et al., "Periodontal regenerative effect of a bovine hydroxyapatite/collagen block in one-wall intrabony defects in dogs: A histometric analysis," Journal of Periodontal \& Implant Science, vol. 41, no. 6, pp. 285-292, 2011.

[32] J. H. Song, H. E. Kim, and H. W. Kim, "Collagen-apatite nanocomposite membranes for guided bone regeneration," Journal of Biomedical Materials Research Part B: Applied Biomaterials, vol. 83, no. 1, pp. 248-257, 2007.

[33] I. Darby, “Periodontal materials," Australian Dental Journal, vol. 56, no. 1, pp. 107-118, 2011. 